\title{
Stress-responsive HILPDA is necessary for thermoregulation during fasting
}

\author{
Matthew J VandeKopple', Jinghai Wu', Lisa A Baer², Naresh C Bal3,a, \\ Santosh K Maurya3,b, Anuradha Kalyanasundaram³, Muthu Periasamy3,b, \\ Kristin I Stanford ${ }^{2}$, Amato J Giaccia ${ }^{4}$, Nicholas C Denko ${ }^{1, c}$ and Ioanna Papandreou1,c \\ 1Department of Radiation Oncology, The Ohio State University, Columbus, Ohio, USA \\ 2Dorothy M. Davis Heart and Lung Research Institute, Department of Physiology and Cell Biology، \\ The Ohio State University, Columbus, Ohio, USA \\ 3Department of Physiology and Cell Biology, The Ohio State University, Columbus, Ohio, USA \\ ${ }^{4}$ Department of Radiation Oncology, Stanford University, Stanford, CA, USA \\ a(N C Bal is now at the School of Biotechnology, KIIT University, Bhubaneswar, India) \\ b(S K Maurya and M Periasamy are now at Sanford Burnham Prebys Medical Discovery Institute at Lake Nona, \\ Orlando, FL, USA) \\ c(N C Denko and I Papandreou contributed equally to this work)
}

Correspondence should be addressed to I Papandreou Email

ioanna.papandreou@ osumc.edu

\begin{abstract}
Hypoxia-inducible lipid droplet-associated protein (HILPDA) has been shown to localize to lipid droplets in nutrient-responsive cell types such as hepatocytes and adipocytes. However, its role in the control of whole-body homeostasis is not known. We sought to measure cell-intrinsic and systemic stress responses in a mouse strain harboring whole-body Hilpda deficiency. We generated a genetically engineered mouse model of whole-body HILPDA deficiency by replacing the coding Hilpda exon with luciferase. We subjected the knockout animals to environmental stresses and measured whole-animal metabolic and behavioral parameters. Brown adipocyte precursors were isolated and differentiated in vitro to quantify the impact of HILPDA ablation in lipid storage and mobilization in these cells. HILPDA-knockout animals are viable and fertile, but show reduced ambulatory activity and oxygen consumption at regular housing conditions. Acclimatization at thermoneutral conditions abolished the phenotypic differences observed at $22^{\circ} \mathrm{C}$. When fasted, HILPDA KO mice are unable to maintain body temperature and become hypothermic at $22^{\circ} \mathrm{C}$, without apparent abnormalities in blood chemistry parameters or tissue triglyceride content. HILPDA expression was upregulated during adipocyte differentiation and activation in vitro; however, it was not required for lipid droplet formation in brown adipocytes. We conclude that HILPDA is necessary for efficient fuel utilization suggesting a homeostatic role for Hilpda in sub-optimal environments.
\end{abstract} Key Words

Journal of Endocrinology (2017) 235, 27-38

\section{Introduction}

Lipid droplets (LDs) are emerging as very dynamic organelles that store lipids and participate in a number of biological processes (Fujimoto \& Parton 2011). LDs consist of a hydrophobic core containing mainly triacylglycerols and steryl esters, surrounded by a phospholipid monolayer that is decorated with proteins (Goodman 2008). It is estimated that 50-200 proteins localize to the LDs surface (Krahmer et al. 2013). http://joe.endocrinology-journals.org DOI: 10.1530/JOE-17-0289
() 2017 Society for Endocrinology Printed in Great Britain
Published by Bioscientifica Ltd 
Some LD-associated proteins are involved in lipid metabolism; however, there are also enzymes and regulatory proteins that do not have recognized functions related to lipids. The LD proteome is very dynamic and is thought to depend on the needs for the cell type- or even LD-type to balance lipid storage and hydrolysis (Hsieh et al. 2012). The most abundant LD proteins belong to the perilipin (PLIN) family of PAT domaincontaining proteins. All five PLINs can coat LDs, and mouse models of Plin deficiency show similar and distinct phenotypes (Sztalryd \& Kimmel 2014). For example, PLIN1 is most highly expressed in white and brown fat and steroidogenic tissues, whereas PLIN2 and PLIN3 are ubiquitously expressed (Sztalryd \& Kimmel 2014). PLIN1 coats the adipocyte LDs and controls basal and stimulated lipolysis. Under basal conditions, it inhibits access of cytosolic lipases to the triacylglycerol core, whereas upon stimulation by $\beta$-adrenergic signaling cAMP levels rise, activated protein kinase A (PKA) phosphorylates PLIN1, which recruits adipose triglyceride lipase and hormonesensitive lipase to LDs, generating maximal lipolysis (Sztalryd et al. 2003, Granneman et al. 2009). In addition to their role in adipocyte biology, LDs have been implicated in foam cell formation in atherosclerosis, hepatitis $\mathrm{C}$ virus replication, liver steatosis and cancer cell survival (Reue 2011, Bensaad et al. 2014, Qiu et al. 2015).

Eutherian mammals have evolved multiple mechanisms to maintain a stable core body temperature. Broadly, these mechanisms minimize heat loss (for example, presence of fur, nesting, changes in body posture) and actively increase heat production. In the initial response to a cold environment, mammals significantly increase their metabolic rate and initiate a shivering response. After prolonged cold exposure, they activate non-shivering thermogenic mechanisms, which result in cold acclimation (Griggio 1982, Cannon \& Nedergaard 2004). Non-shivering thermogenesis depends on heat generation by both brown adipose tissue (BAT) and skeletal muscle (Silva 2011). BAT is characterized by the presence of the mitochondrial UCP1 protein, which uncouples respiratory chain electron transfer from ATP production. This futile cycle dissipates energy as heat and is stimulated by $\beta$-adrenergic stimulation and activation of lipolysis (Cannon \& Nedergaard 2011). Additionally, non-shivering muscle-based thermogenesis is also known to participate in cold- and diet-induced heat generation, particularly when there is diminished BAT-dependent thermogenesis. For example, Sarcolipin, a regulator of the SERCA (sarcoendoplasmic reticulum (SR) calcium transport
ATPase) pump has been shown to promote a futile cycle of ATP hydrolysis by uncoupling ATP hydrolysis from $\mathrm{Ca}^{2+}$ pumping back into the $\mathrm{SR}$, resulting in heat production and protection from diet-induced obesity (Smith et al. 2002, Bal et al. 2012, Maurya et al. 2015).

Hypoxia-inducible lipid droplet associated protein (HILPDA) was originally identified as a hypoxia-inducible gene and was named hypoxia-inducible gene 2 (HIG2) (Denko et al. 2000). Later studies confirmed its hypoxic inducibility in different cell contexts (Wang et al. 2005, Elvidge et al. 2006). Evolutionary appearance of Hilpda is quite recent, as orthologs of the human gene product are limited to those in other mammals. The lipid droplet localization of Hilpda was determined by Gimm and coworkers who also showed that its overexpression increases the intracellular accumulation of neutral lipids and cytokine expression in tumor cells (Gimm et al. 2010). Evidence for the role of Hilpda in organismal physiology has recently emerged through the use of tissuespecific deletion in engineered mouse strains. Genetic manipulation in murine liver has implicated HILPDA in triglyceride accumulation by mechanisms involving PPAR $\alpha$-dependent Hilpda induction, triglyceride secretion and regulation of lipolysis, although direct inhibition of lipase activity was not found (Mattijssen et al. 2014, DiStefano et al. 2015). Additionally, deletion of Hilpda in WAT and/or BAT did not impact lipolysis, despite its robust induction during adipocyte differentiation in vitro and nutritional manipulations in vivo (DiStefano et al. 2016, Dijk et al. 2017).

In this work, we investigated the impact of wholebody Hilpda loss on survival, growth and adaptation to environmental stresses. Using a novel genetically engineered mouse model, we have uncovered Hilpda's involvement in thermoregulation and energy expenditure by exposing the animals in stressful environments similar to what would be found outside the climate- and diet-controlled laboratory.

\section{Materials and methods}

\section{Mouse genetics}

A mutant mouse strain where the Hilpda gene was knocked out and firefly luciferase was knocked in was generated by Xenogen Biosciences (Cranbury, NJ, USA). BAC clone RP23-104P4 was used for generating homologous arms and southern probes by PCR or RED cloning/gap-repair method. The final vector was obtained
(C) 2017 Society for Endocrinology Printed in Great Britain
Published by Bioscientifica Ltd 
by standard molecular cloning methods and linearized before electroporation into C57BL/6-Tac ES cells. The targeted ES cells were injected into FVB blastocysts and chimeras were mated to WT C57BL/6-Tac females. We received mice at N3, F5 and back-crossed them sequentially to both C57BL/6-Tac and C57BL/6J animals for a total of six generations. For genotyping, we used a triplex PCR with primers: 53F:5'-CCTGGGCTACATAGCAGGGAGAGG-3', KOR: 5'-GATGCCCAGCACATAGAGGTTCAGC-3' and Luc-R: 5'-CTCCAGCGGTTCCATCTTCCAGC-3'. Mouse chow was from Teklad (\#LM-485), with the following caloric composition: carbohydrates $58 \%$, protein $25 \%$ and fat $17 \%$. Killing was performed by $\mathrm{CO}_{2}$ inhalation followed by cervical dislocation. All animal studies were approved by the Institutional Animal Care and Use Committee (IACUC) of The Ohio State University, in accordance with the National Institutes of Health Guide for the Care and Use of Laboratory Animals.

\section{Metabolic cage measurements}

Whole animal metabolic parameters were measured using the Comprehensive Lab Animal Monitoring System (CLAMS) equipped with a temperature-controlled environmental chamber from Columbus Instruments (Columbus, OH, USA). Female, single-housed 4- to 5 -month-old mice were used for all experiments, unless otherwise indicated. For the work done at $22^{\circ} \mathrm{C}$, the measurements of the first $24 \mathrm{~h}$ were excluded from the analysis to allow for acclimation to the new environment. For the experiments performed at $30^{\circ} \mathrm{C}$, the first $48-60 \mathrm{~h}$ were excluded in order for brown fat thermogenesis to cease. For body temperature measurements, thermal transponders (IPTT300, Bio Medic Data System, Seaford, DE, USA) were implanted under $2 \%$ isofluorane anesthesia subdermally in the interscapular region, attempting to place the transponder directly above the BAT. Readings were recorded non-invasively as previously described (Bal et al. 2012). The procedure was minimally invasive and the animals resumed normal activity immediately after recovery from anesthesia.

\section{Blood and tissue chemistry}

To test for glucose tolerance, mice were fasted overnight and $2 \mathrm{~g} / \mathrm{kg}$ glucose in saline was injected intraperitoneally. Blood was drawn from the tail at 0, 15, 30, 60 and $90 \mathrm{~min}$ and tested with a ReliOn Ultima glucose meter (Walmart, Bentonville, AR, USA). Fasted serum chemistry analysis was performed by the OSU Comparative Pathology and Phenotyping core facility. Tissue triglyceride content was measured with the Triglyceride Quantification Kit (Abcam) following the manufacturer's instructions.

\section{In vitro adipocyte differentiation}

Stromal vascular fraction (SVF) from interscapular BAT was isolated from 3 WT and 3 Hilpda KO mice between the ages of 4-5 weeks. The isolation buffer contained $123 \mathrm{mM} \mathrm{NaCl}$, $5 \mathrm{mM} \mathrm{KCl}, 1.3 \mathrm{mM} \mathrm{CaCl}_{2}, 5 \mathrm{mM}$ glucose, $100 \mathrm{mM}$ HEPES, $4 \%$ BSA, $1.5 \mathrm{mg} / \mathrm{mL}$ collagenase A (Sigma), $100 \mathrm{U} / \mathrm{mL}$ penicillin and $100 \mu \mathrm{g} / \mathrm{mL}$ streptomycin. Cells were expanded in DMEM supplemented with 15\% FBS and were used within two passages. For SVF differentiation into brown adipocytes, $25 \times 10^{4}$ undifferentiated cells were plated in six-well plates and grown to confluence. The following day, growth media was changed to differentiation induction media consisting of DMEM with $10 \%$ FBS, $20 \mathrm{nM}$ insulin, $1 \mathrm{nM} \mathrm{T3}, 125 \mu \mathrm{M}$ indomethacin, $2 \mu \mathrm{g} / \mathrm{mL}$ dexamethasone and $0.5 \mathrm{mM}$ IBMX (all from Sigma). Two days later, the induction media were replaced by differentiation maintenance media of DMEM, 10\% FBS, $20 \mathrm{nM}$ insulin and $1 \mathrm{nM}$ T3 for an additional 4-6 days.

\section{Adipocyte metabolic assays}

Brown adipocyte oxygen consumption and mitochondrial function were analyzed in an XF96 Seahorse analyzer (Agilent). Three independent SVF preparations per genotype were plated onto L-lysine-coated XF96 plates in five replicates and differentiated for 6 days as described in the 'In vitro adipocyte differentiation' section above. Basal oxygen consumption rates (OCR) were measured in maintenance media and uncoupled oxygen consumption was calculated $15 \mathrm{~min}$ after the injection of $10 \mu \mathrm{M}$ forskolin and $10 \mu \mathrm{M}$ oligomycin. For glycerol release quantification, day 7 differentiated adipocytes were switched to FBS-free maintenance media for $2 \mathrm{~h}$ and glycerol concentration in the media was measured with a Glycerol Assay Kit (Sigma). The cell monolayers were lysed in protein lysis buffer, and total protein content was used to normalize the glycerol release values.

\section{Establishment and treatment of MEF cell lines}

Embryos from Hilpda Het $\times$ Het crosses were isolated at 13.5 p.c., and the heads and internal organs were removed and used for genotyping. Fibroblasts were isolated by

Published by Bioscientifica Ltd. 
mincing in $0.25 \%$ trypsin/EDTA solution. Immortalized cell lines were established by retroviral infection with a pBABE-puro SV40 LT plasmid (from Thomas Roberts through Addgene \#13970) and puromycin resistance selection. In vitro hypoxia was achieved in an H35 HypOxystation (HypOxygen, Frederick, MD, USA).

\section{Western blotting}

Cell monolayers and mouse organs were washed in PBS and lysed in radioimmunoprecipitation assay buffer, sonicated and cleared by centrifugation. Protein concentration was measured with a bicinchoninic acid assay kit (ThermoFisher). Proteins were separated on Tris-glycine or Tris-tricine gels, transferred onto PVDF membranes and probed with the following primary antibodies: anti-CEBPb (Cell Signaling), anti-PPARg and anti-Plin1 (Thermo Scientific), anti-pyruvate dehydrogenase E1a (Abcam), anti-tubulin (Santa Cruz) or a custom-made antiHilpda polyclonal antibody raised against the C-terminus of human Hilpda (Open Biosystems, Huntsville, AL, USA). Primary antibodies were detected with IRDye 680RD and IRDye $800 \mathrm{CW}$ goat anti-mouse and anti-rabbit secondary antibodies (LI-COR, Lincoln, NE, USA), respectively, and infrared signal was detected with an Odyssey SA LI-COR imager.

\section{Bioluminescent imaging}

Mice were injected intraperitoneally with $150 \mathrm{mg} / \mathrm{kg}$ potassium d-luciferin (PerkinElmer) in saline and killed 10 min later. Organs were quickly removed and imaged in a Xenogen IVIS100 system (PerkinElmer).

\section{Gene expression profiling}

Gene expression analysis was performed using RNA from interscapular brown fat of overnight fasted mice $(n=4)$. Briefly, RNA was isolated with TRIzol (Thermo Scientific). The samples were biotin labeled and hybridized to Affymetrix GeneChip Mouse Transcriptome Array 1.0 platform by the Genomics Shared Resource Facility, The Ohio State University Comprehensive Cancer Center. Normalizations and gene expression changes were analyzed in the Affymetrix Expression Console. The microarray data are deposited with the Gene Expression Omnibus (GEO) with accession number GSE100201.

\section{Statistical analysis}

Data were analyzed with GraphPad Prism 7 (GraphPad Software). Data from two groups only were analyzed by unpaired Student's $t$-test. Metabolic data were analyzed by two-way ANOVA and Fisher's LSD test. Statistical significance was set at $P<0.05$ and data are presented as mean + S.E.M. The number of biological replicates or sample size is indicated in the respective figure legends.

\section{Results}

\section{Establishment of Hilpda KO mice}

The mouse Hilpda gene contains two exons and is predicted to code for two transcript variants. Our Hilpda $\mathrm{KO}$ /reporter strain (hereafter referred to as $\mathrm{KO}$ ) was generated by replacing the coding sequence of exon 2 with the firefly luciferase coding sequence (Fig. 1A and B). The KO mice are viable, fertile and born at the expected Mendelian ratios (data not shown). To map Hilpda promoter activity in vivo, we injected adult $\mathrm{KO}$ mice with D-luciferin, killed them and imaged excised tissues for bioluminescence (Fig. 1C). High luciferase activity was detected in a large number of organs and tissues, including lung, white adipose tissue, liver and intestine. This suggests constitutive promoter activity in organs of diverse functions and/or high activity in stromal components. Western blotting on WT organ lysates showed variable levels of expression, with the highest levels in lung followed by the heart and liver (Fig. 1D). To validate the specificity of the polyclonal anti-Hilpda antibody, we treated immortalized MEFs from WT and $\mathrm{KO}$ embryos with normoxia or $1 \% \mathrm{O}_{2}$ hypoxia for $24 \mathrm{~h}$. We found significant hypoxic induction of Hilpda in the WT cells and confirmed the complete loss of detectable protein in the KO samples (Fig. 1E). Hilpda loss did not impact body weight gain in female mice fed a standard diet (Fig. 1F); however, daily food consumption was decreased in the KO animals (Fig. 1G). This decreased food intake, associated with normal weight gain, raised the possibility of either lower energy expenditure or abnormal nutrient absorption in the absence of Hilpda.

\section{Altered metabolism in the absence of HILPDA under chronic, mild cold stress}

To characterize possible metabolic alterations by the complete genetic ablation of Hilpda, we measured basal

Published by Bioscientifica Ltd 
A

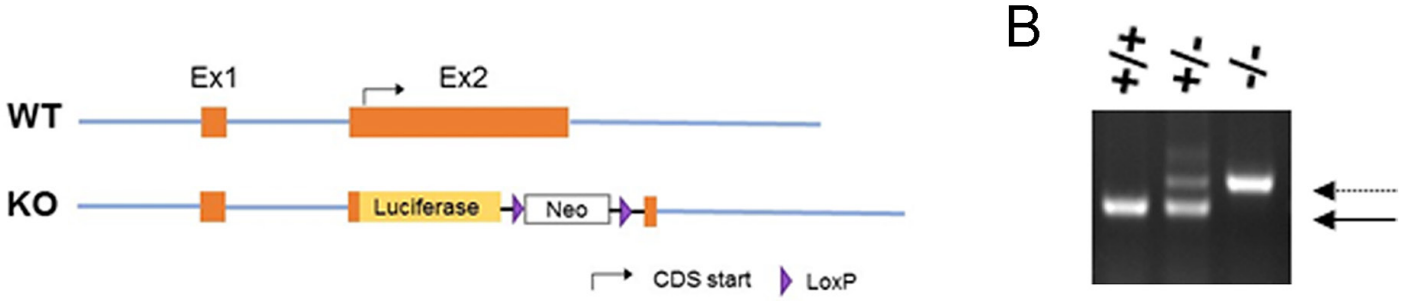

C
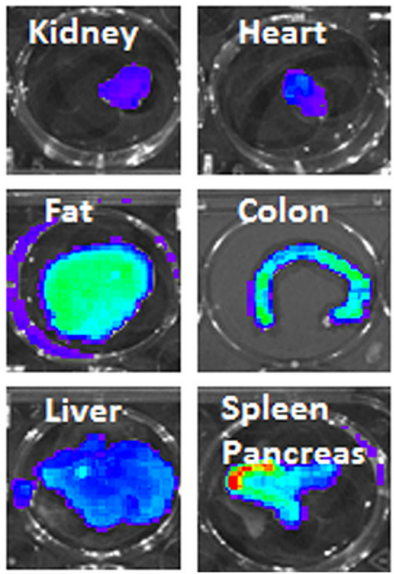

F

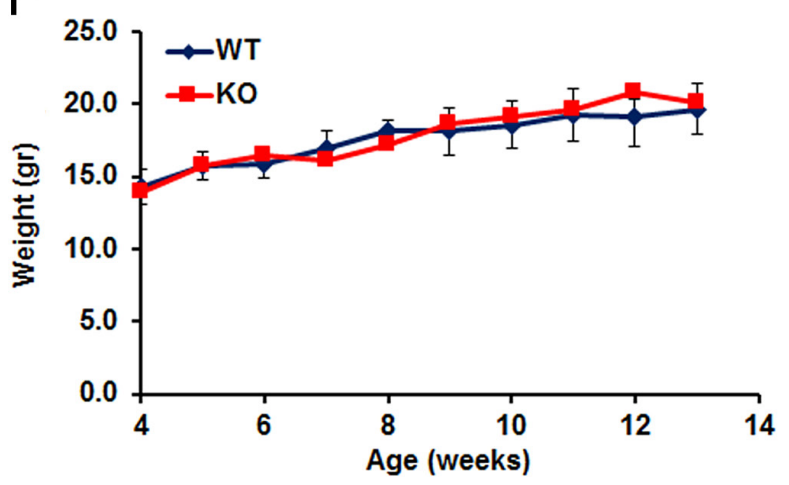

D
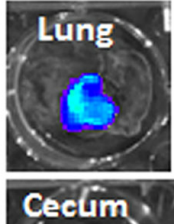

E
Tubulin
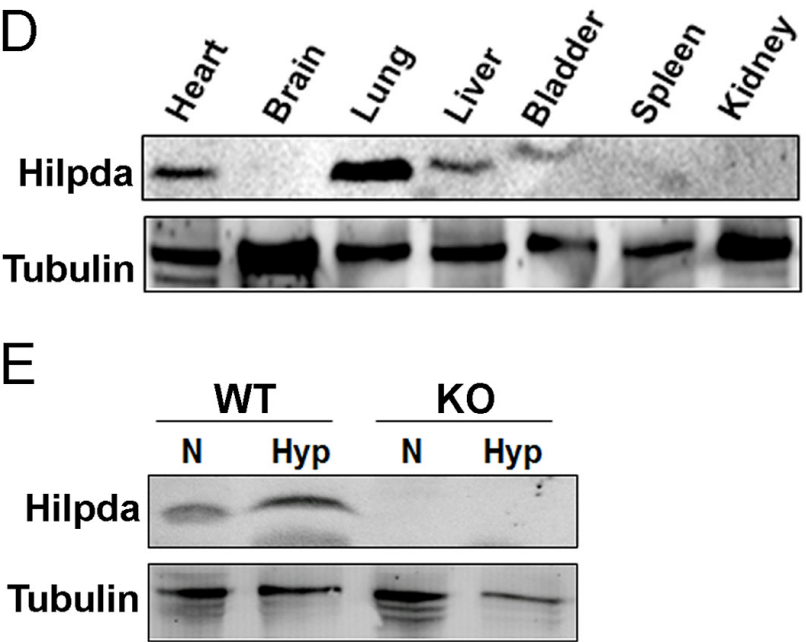

G

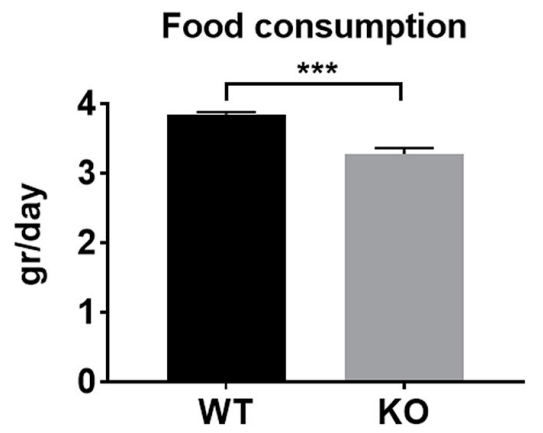

Figure 1

Generation of Hilpda KO mice. (A) Schematic of the murine Hilpda gene and the designed knockout/reporter allele. (B) Representative image of genotyping PCR products of Hilpda WT (+/+), heterozygous (+/-) and KO (-/-) animals. Solid arrow: wild type product $0.32 \mathrm{~kb}$, dashed arrow: knockout product $0.39 \mathrm{~kb}$. (C) Bioluminescent detection of Hilpda promoter activity in a $\mathrm{KO}$ mouse after injection of $0.1 \mathrm{mg} / \mathrm{mouse} \mathrm{D}$-luciferin followed by killing $10 \mathrm{~min}$ later. (D) Hilpda protein expression in the indicated organs of a fed female mouse. (E) Immortalized mouse embryonic fibroblasts were treated in vitro with normoxia $(\mathrm{N})$ or $1 \% \mathrm{O}_{2}(\mathrm{Hyp})$ for $24 \mathrm{~h}$ and Hilpda protein levels were detected by western blotting. (F) Body weight curves of group housed female mice between the ages of 4 and 13 weeks $(n=7)$. (G) Daily food consumption of single-housed female mice $(n=5)$ ( $* * * P<0.0001$ by Student's $t$-test).

metabolic parameters of adult, female mice maintained in standard housing temperatures $\left(22^{\circ} \mathrm{C}\right)$. The $\mathrm{KO}$ animals had lower oxygen consumption, voluntary physical activity, as well as energy expenditure throughout the light cycle (Fig. 2A, B and C). Further analysis of our metabolic cage data showed that the significantly lower total activity in the KO mice was attributed to both lower ambulatory activity in the $\mathrm{X}$ plane and lower $\mathrm{Z}$ plane breaks (rearing activity) (data not shown). We did not measure appreciable changes in the respiratory exchange ratio (RER) (Fig. 2D). This indicates an overall reduced metabolism without a shift in the relative contribution of carbohydrate and fat oxidation.

\section{Thermoneutrality abolishes the Hilpda KO phenotype}

The reduced oxygen consumption at $22^{\circ} \mathrm{C}$ in conjunction with reduced ambulatory activity in the Hilpda $\mathrm{KO}$ mice raised the possibility of an adaptive change in the 

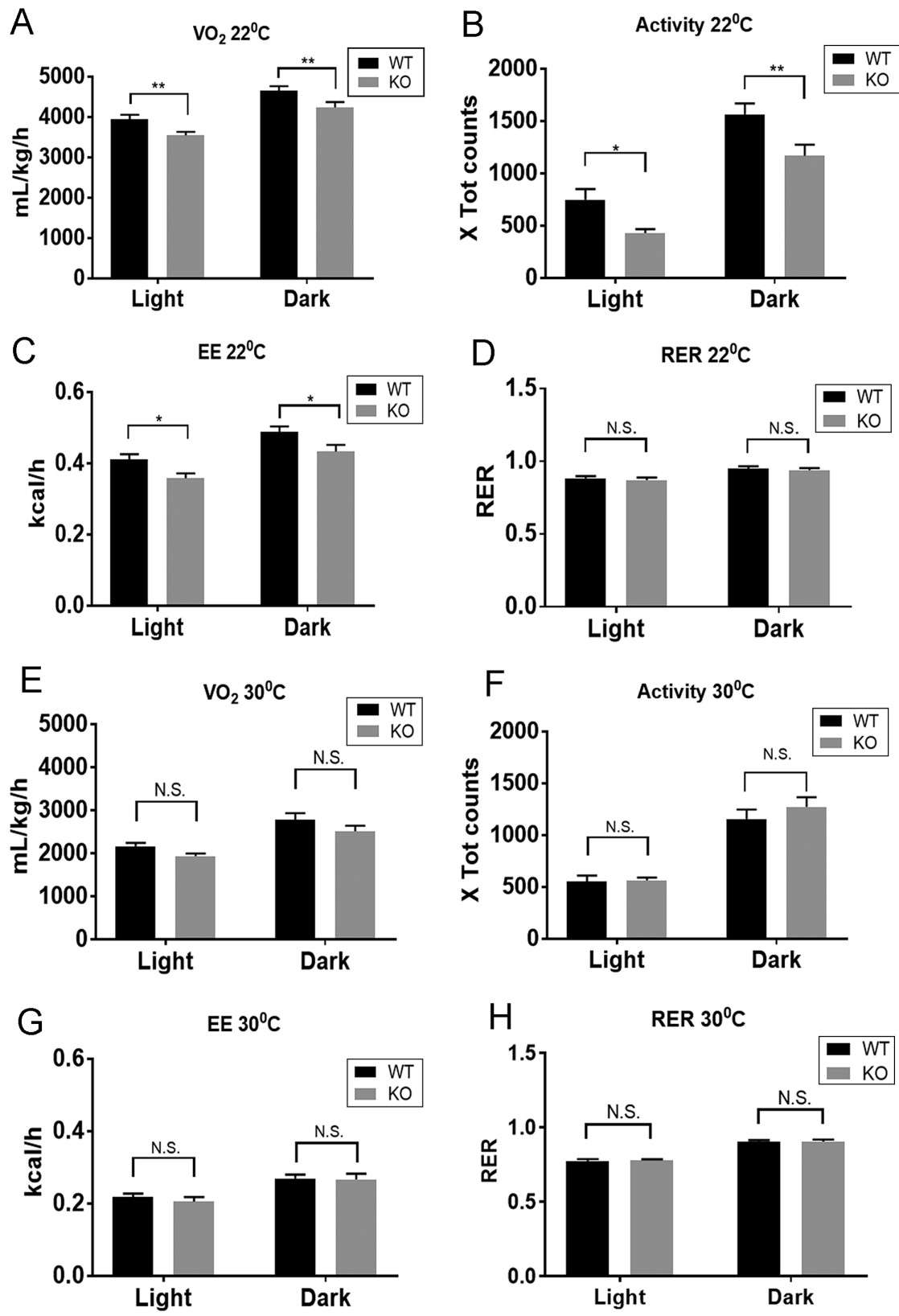

Figure 2

Hilpda ablation reduces whole metabolism at $22^{\circ} \mathrm{C}$ but not at $30^{\circ} \mathrm{C}$. (A, B, C and D) Metabolic parameters of individually housed, fed, female 4- to 5-month-old mice were measured in metabolic cages following a 24-h acclimation period. (A) Average $\mathrm{VO}_{2}$ consumption, (B) physical activity, (C) energy expenditure, (D) respiratory exchange ratio $(n=16)$. (E, F, G and H) Metabolic parameter analysis of fed female mice at thermoneutrality following 48- to 60-h acclimation. (E) Average $\mathrm{VO}_{2}$ consumption, (F) physical activity, (G) energy expenditure, (H) respiratory exchange ratio $(n=12-15)$. Light: 7:00-19:00, dark: 19:00-7:00. $\left({ }^{*} P<0.05\right.$, ${ }^{*} P<0.01$, N.S. not significant by two-way ANOVA and Fisher's LSD test). Error bars: S.E.M.

relative contribution of brown fat and muscle toward the maintenance of normal body temperature. In order to dissociate the two, we acclimatized WT and KO animals to thermoneutrality $\left(30^{\circ} \mathrm{C}\right)$ to minimize BAT activation and performed indirect calorimetry (Fig. 2E, F, G and H). As expected, average oxygen consumption and energy expenditure of both genotypes was lower compared to $22^{\circ} \mathrm{C}$, since the energetic requirement for heat production was minimal. Interestingly, thermoneutrality eliminated the differences in oxygen consumption and in physical activity seen at regular housing temperatures, suggesting that Hilpda participates in homeostatic mechanisms at ambient temperatures.

\section{Hilpda is upregulated early during differentiation} in vitro

Previous reports have shown that Hilpda is expressed in human and murine adipocytes and responds to PPAR- and b-adrenergic signaling (DiStefano et al. 2016, Dijk et al. 2017). To determine the regulation of Hilpda expression and its impact on adipocyte differentiation in our genetically engineered model, we isolated the stromal vascular fraction (SVF) from interscapular BAT and differentiated it in vitro (Fig. 3). First, we followed the changes in protein levels of known transcriptional regulators and adipocyte markers over time, in order 
A

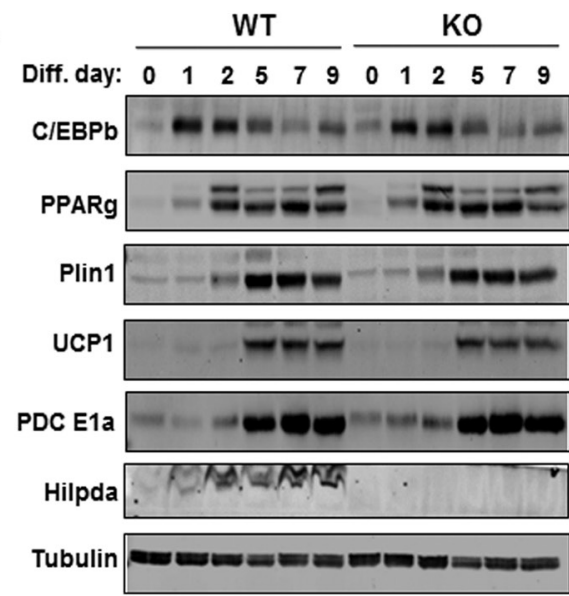

B

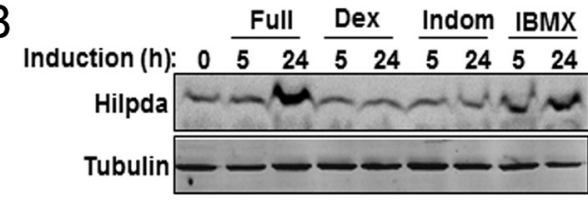

C

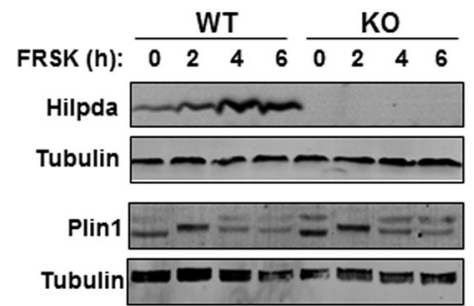

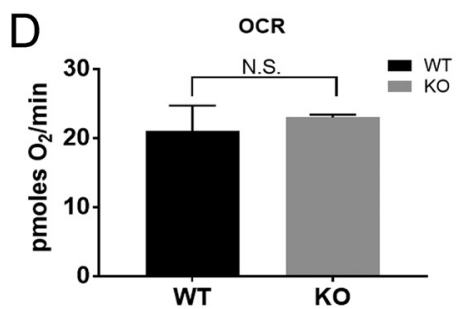

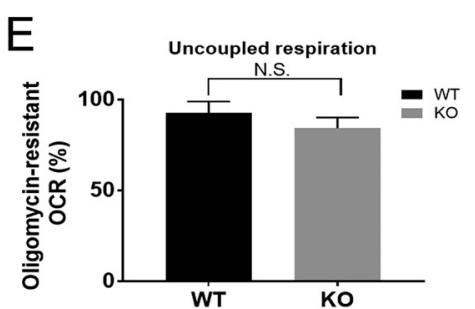

$\mathrm{F}$

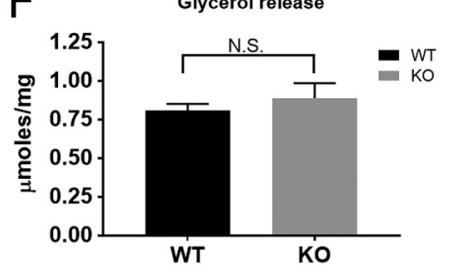

Figure 3

Upregulation of Hildpa during BAT

differentiation and activation in vitro. (A) Time course of adipocyte markers' protein levels during the differentiation of stromal vascular fraction isolated from the interscapular brown fat of 4-week-old mice. Day 0 corresponds to confluent cell cultures before the addition of the induction cocktail. Representative images of two independent SVF preparations are shown. (B) Western blot of Hilpda protein in early time points during differentiation. Day 0 preadipocytes were incubated in BAT maintenance media plus the complete induction cocktail $(2 \mu \mathrm{g} / \mathrm{mL}$ dexamethasone, $125 \mu \mathrm{M}$ indomethacin, $0.5 \mathrm{mM}$ IBMX) or maintenance media plus individual inducers for 5 or $24 \mathrm{~h}$. (C) Protein levels of Hilpda and Plin 1 on day 7 brown adipocytes after $10 \mu \mathrm{M}$ forskolin treatment for the indicated time points. (D, E and F) Brown adipocyte metabolism in vitro: (D) oxygen consumption rates. (E) Proton leak as determined by oxygen consumption resistant to $10 \mu \mathrm{M}$ forskolin/oligomycin $(n=3)$. (F) Basal glycerol release per mg of protein. Adipocytes were switched to serum-free media for $2 \mathrm{~h}$ and the supernatant was analyzed for glycerol content. The cell monolayer was lysed in RIPA buffer and total protein content was used for normalizations $(n=3)$. to reveal any possible impact of Hilpda ablation on the differentiation cascade (Fig. 3A). We detected early and transient upregulation of $\mathrm{CEBP} / \mathrm{b}$ by day 1 in both genotypes, followed by the appearance of PPARg and at later time points increases in Plin1, UCP1 and the E1a subunit of the mitochondrial enzyme pyruvate dehydrogenase also in both genotypes. We concluded that the hormonal response, adipogenic program and expression of brown fat-specific markers, like mitochondrial biogenesis and UCP1 expression, are not impaired in the Hilpda KO cells. Interestingly, we observed upregulation of Hilpda protein early in the differentiation course, with detectable levels already on day 1 . These kinetics differ significantly from those of Plin1 and suggest regulatory mechanisms in addition to the response to PPAR.

Based on the upregulation of Hilpda during the induction phase of differentiation, we hypothesized that one or more components of the chemical cocktail were responsible for this acute response. We performed a time course on day 0 preadipocytes incubated either in the full cocktail (insulin, T3, dexamethasone, indomethacin, IBMX) or BAT maintenance media (insulin and T3) plus the individual induction components and found that IBMX was sufficient to increase HILPDA protein (Fig. 3B). IBMX is a non-specific phosphodiesterase inhibitor, suggesting that elevation in cyclic nucleotide levels positively regulate HILPDA. Moreover, when day 7 adipocytes were activated with $10 \mu \mathrm{M}$ forskolin to mimic $\beta$-adrenergic signaling, we observed an additional accumulation of HILPDA (Fig. 3C), further supporting its regulation by cAMP. We also probed forskolin-treated samples for Plin1 as a control for forskolin's activity, which caused a shift in the electrophoretic mobility of Plin1. This is consistent with the phosphorylation of Plin1 by PKA during the stimulation of lipolysis (Greenberg et al. 1991).

\section{Hilpda KO brown adipocytes are functional}

Next, we performed a functional characterization of Hilpda WT (wild type) and $\mathrm{KO}$ adipocytes by measuring metabolic parameters. OCR, degree of uncoupled mitochondrial oxygen consumption, as well as basal glycerol release rates was similar between the groups, showing that Hilpda is not necessary for mitochondrial fuel oxidation 
or triglyceride hydrolysis, at least in the presence of high levels of nutrients (Fig. 3D, E and F). Consistent with our in vitro findings, Hilpda $\mathrm{KO}$ mice were able to increase their metabolism compared to ambient temperature (Fig. 4A, B, $\mathrm{C}$ and D) and defend body temperature when exposed to cold acutely for $6 \mathrm{~h}$ (Fig. 4E). Interestingly, following the stress, the KO mice increased their food intake compared to the WT mice (Fig. 4F).

\section{Hilpda participates in thermoregulation during fasting}

The ability of the Hilpda $\mathrm{KO}$ mice to thermoregulate in the fed state prompted us to introduce an additional stressor and subject them to fasting, another physiologic condition that induces lipid store mobilization and systemic changes in metabolism. Following food deprivation during the dark phase for $12 \mathrm{~h}$ at $22^{\circ} \mathrm{C}$, the KO mice had significantly lower body temperature than the WT (Fig. 5A). The defect became more severe when the fasting was followed by exposure to cold (Fig. 5B). To identify genes whose expression may be deregulated in the absence of Hilpda and could be responsible for this phenotype, we performed microarray gene expression analysis on brown fat from fasted mice. Statistical analysis of the data using as cutoff values a two-fold difference between genotypes, with $P<0.01$ and FDR 0.05 did not yield any candidates regulated by the Hilpda status. A curated list of select genes associated with BAT function is shown in Table 1. This result leads us to conclude that the hypothermia of the Hilpda KO animals cannot be traced to gene expression changes in the BAT.

Liver- and adipose-specific Hilpda deletion has been reported to improve glucose tolerance (DiStefano et al. $2015,2016)$. The glucose tolerance test after $16 \mathrm{~h}$ of overnight fasting in our whole-body $\mathrm{KO}$ model showed a trend toward higher glucose peak levels in the absence of Hilpda but it did not reach statistical significance (Fig. 5C). We performed fasting serum lipid analysis to test if Hilpda loss would alter systemic lipid mobilization, either as lipid release or as uptake to and from the bloodstream and found higher levels of HDL in the $\mathrm{KO}$ mice, while triglycerides, cholesterol, LDL, CK and LDH levels were unaltered (Table 2). Additionally, we measured the animals' metabolism during fasting (Fig. 6A, B, C and D). Oxygen consumption and energy expenditure were lower in the KO group during the active phase of the daily
A
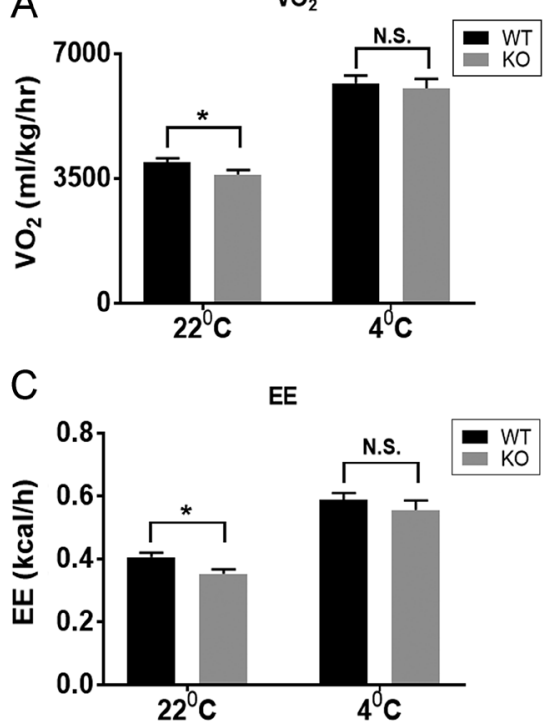

E
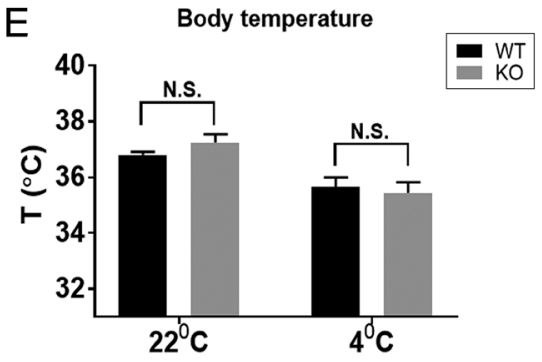

B

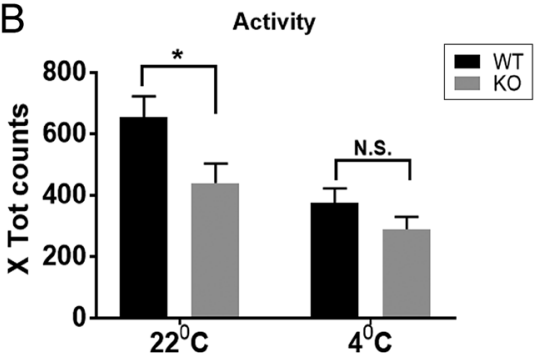

D

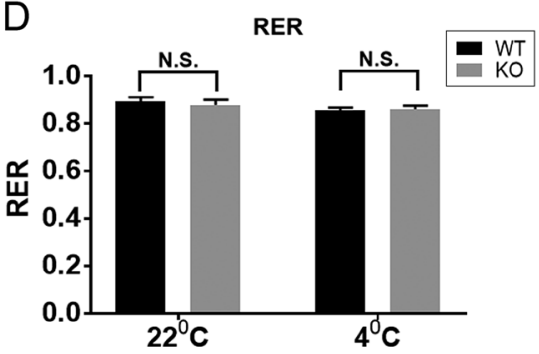

$\mathrm{F}$

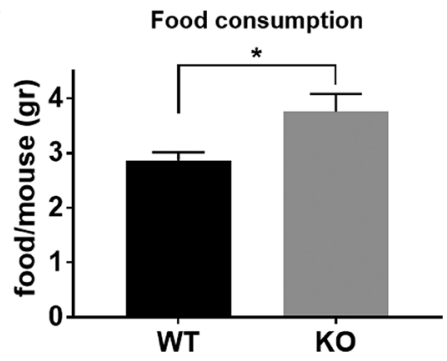

Figure 4

Metabolic parameters of fed, female mice during acute cold exposure. Animals were acclimatized in the metabolic cages at $22^{\circ} \mathrm{C}$ for $48 \mathrm{~h}$ and the temperature was dropped to $4^{\circ} \mathrm{C}$ during the light cycle for $6 \mathrm{~h}$. The $22^{\circ} \mathrm{C}$ data correspond to $6 \mathrm{~h}$ of the light cycle on the day before the cold exposure. (A) Average $\mathrm{VO}_{2}$ consumption, (B) physical activity, (C) energy expenditure, (D) respiratory exchange ratio $(n=11-13)$. (E) Body temperature measured by implanted thermotransponders at the beginning and the end of cold exposure $(n=5)$. (F) food consumption for the first $24 \mathrm{~h}$ after the end of the cold exposure $(n=5)$. Error bars: S.E.M. $\left({ }^{*} P<0.05\right.$, N.S: not significant by Student's $t$-test). 

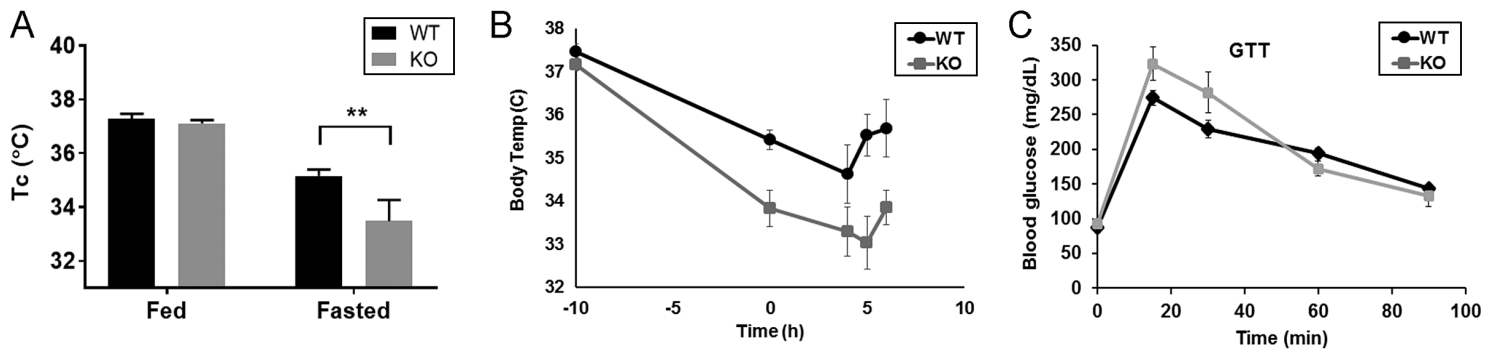

Figure 5

Hilpda-dependent responses to fasting. (A) 4- to 5-month-old female mice were implanted subdermally in the interscapular area with thermotransponders and recovered for at least 4 days. Food was removed in the evening and temperatures were recorded 12h later ( $n=14-16)$. (B) Mice implanted with thermotransponders were fasted as described above during the dark phase at $22^{\circ} \mathrm{C}$, and the following morning, the temperature was dropped to $4^{\circ} \mathrm{C}(\mathrm{t}=0$ on the graph) for an additional $6 \mathrm{~h}$. $(n=8)$ (C) Glucose tolerance test after an overnight fast and intraperitoneal injection of $2 \mathrm{~g} / \mathrm{kg}$ glucose $(n=7)$.

cycle and physical activity was decreased throughout the experiment. The respiratory exchange ratio was not different between the groups similar to what we had observed in the fed state. Finally, triglyceride content in WAT, liver and gastrocnemius muscles of fasted mice was not affected by the Hilpda status (Fig. 6E, F and G).

\section{Discussion}

Here, we present the generation and metabolic characteristics of a Hilpda whole animal knockout mouse model. We found that Hilpda is not required for viability, growth and reproduction. At standard housing temperatures, the Hilpda $\mathrm{KO}$ mice had reduced metabolism, consuming less oxygen, being less active and expending less energy. Concomitantly, their food intake was lower, a finding that can explain their normal weight gain. Our result that thermoneutrality diminishes these metabolic differences suggests that thermoregulatory mechanisms have undergone adaptations in the absence of HILPDA. Our data also suggest that if any of these adaptations involve the BAT, they do not include changes

Table 1 Log2-transformed expression levels of representative genes in Hilpda WT and KO BAT of overnight fasted mice $(n=4)$.

\begin{tabular}{lccc}
\hline Gene & Hilpda WT (log2 aver. signal) & Hilpda KO (log2 aver. signal) \\
\cline { 1 - 1 } Plin1 & 15.76 & 14.89 \\
Plin2 & 17.49 & 17.05 \\
Pparg & 16.17 & 16.06 \\
Ucp1 & 19.93 & 19.93 \\
Ppargc1a & 13.60 & 13.91 \\
Ppargc1b & 10.94 & 10.43 \\
Lipe & 15.39 & 15.03 \\
Pnpla2 & 19.56 & 19.55 \\
Adrb3 & 9.47 & 9.06 \\
\hline
\end{tabular}

at the level of gene transcription. This is in agreement with previous reports who were unable to find significant gene expression changes in tissue-specific Hilpda $\mathrm{KO}$ models (Mattijssen et al. 2014, DiStefano et al. 2015).

It is possible that the primary impact of Hilpda is on locomotor activity, either at the level of muscle contractility or by other neurological, behavioral or hormonal mechanism(s). These may, for example, include compensations such as change in body posture and blood flow or changes in skeletal muscle thermogenesis through uncoupling of the SERCA pump by Sarcolipin (Rowland et al. 2015). It is also possible that, in our genetic background, BAT function below thermoneutrality is altered when Hilpda is absent, similar to what has been seen in the adipose tissue-specific KO model (DiStefano et al. 2016, Dijk et al. 2017) In agreement with recently published reports, we did not find a cell-autonomous defect of HILPDA KO adipocytes in their differentiation capacity or lipolysis (DiStefano et al. 2016, Dijk et al. 2017). Hilpda was, however, required for body temperature defense after fasting, and the hypothermia of the KO mice was associated with rebound hyperphagia. Adipose tissue-specific and also liver-specific deletion of Hilpda has been shown to improve glucose tolerance in male mice (DiStefano et al. 2015). Females from the whole-body KO strain did not show a similar phenotype, and fasting liver triglyceride content was not significantly decreased. Although the possibility of gender-, sub-strain- or dietspecific phenotypes cannot be ruled out, our results raise the possibility that the simultaneous deletion of Hilpda in all tissues re-equilibrates lipid droplet trafficking.

Hilpda's first identified gene expression inducer was hypoxia (Denko et al. 2000), a finding that we confirmed here in immortalized MEFs. It is now evident that additional stresses and transcription factors, such as PPARs, cAMP and nutrient imbalance induce Hilpda

Published by Bioscientifica Ltd. 
Table 2 Fasted serum parameters of female Hilpda WT and KO mice $(n=5)$. $P$ value: Student's unpaired two-tailed $t$-test ( $\left.{ }^{*}<0.05\right)$.

Cholesterol (mg/dL)
Creatine kinase (U/L)
$\mathrm{HDL}(\mathrm{mg} / \mathrm{dL})$
$\mathrm{LDL}(\mathrm{mg} / \mathrm{dL})$
Triglycerides (mg/dL)
Lactate dehydrogenase (U/L)

\begin{tabular}{c}
\hline $\mathbf{W T}($ mean \pm s.E.M. $)$ \\
$79.9 \pm 3.4$ \\
$508.2 \pm 26.0$ \\
$29.8 \pm 2.9$ \\
$35.6 \pm 3.6$ \\
$71.4 \pm 5.7$ \\
$205.8 \pm 34.4$
\end{tabular}

\begin{tabular}{c}
\hline KO $($ mean \pm S.E.M. $)$ \\
$86.1 \pm 4.2$ \\
$859.4 \pm 169.7$ \\
$38.8 \pm 1.6$ \\
$34.6 \pm 2.3$ \\
$62.8 \pm 3.4$ \\
$261.8 \pm 41.1$
\end{tabular}

\begin{tabular}{c|}
\hline $\boldsymbol{P}$ value \\
\hline 0.27 \\
0.07 \\
$0.02^{*}$ \\
0.82 \\
0.23 \\
0.32 \\
\hline
\end{tabular}

expression (this work) (Gimm et al. 2010, Wang et al. 2010, Mattijssen et al. 2014, DiStefano et al. 2015, 2016, Dijk et al. 2017). The mechanistic details of Hilpda-dependent lipid accumulation are not known yet and require additional biochemical investigation. An intriguing characteristic of Hilpda is the lack of any significant similarity between this short 63-64aa protein and other more ancient proteins, despite the presence of lipid storage organelles in all eukaryotes. Based on this paucity of annotated functional domains, we propose that Hilpda functions as a docking site or as a regulatory component of larger complex(es) that control lipid droplet dynamics. Furthermore, the implication of Hilpda in thermoregulation, given the relatively recent evolutionary emergence of the gene, makes it reasonable to propose that Hilpda serves to enhance some unique aspects of the mammalian fitness that are absent from other classes in the animal kingdom.

Emerging evidence shows that more than one mechanism, possibly via different protein interactions, may dictate complementary routes to the end point of lipid droplet growth. In support of this model, Hilpda has been reported to negatively regulate lipolysis and triglyceride secretion in the liver, but not in adipose tissue (Mattijssen et al. 2014, DiStefano et al. 2015, 2016, Dijk et al. 2017). Cell context and the particular lipid droplet proteome may also determine distinct ways of lipid ester storage dynamics (Beller et al. 2008, Guo et al. 2008, Khor et al. 2014) and therefore the significance of Hilpda in these processes.

In conclusion, we found that genetic deletion of Hilpda decreases physical activity and energy expenditure and sensitizes mice to fasting-induced hypothermia.
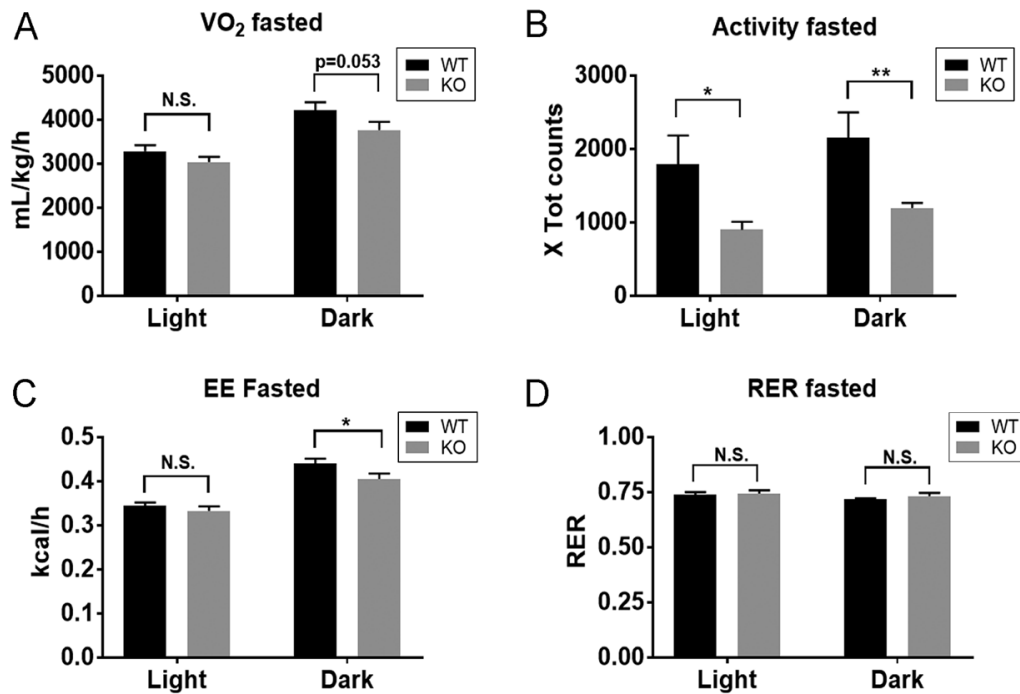

D
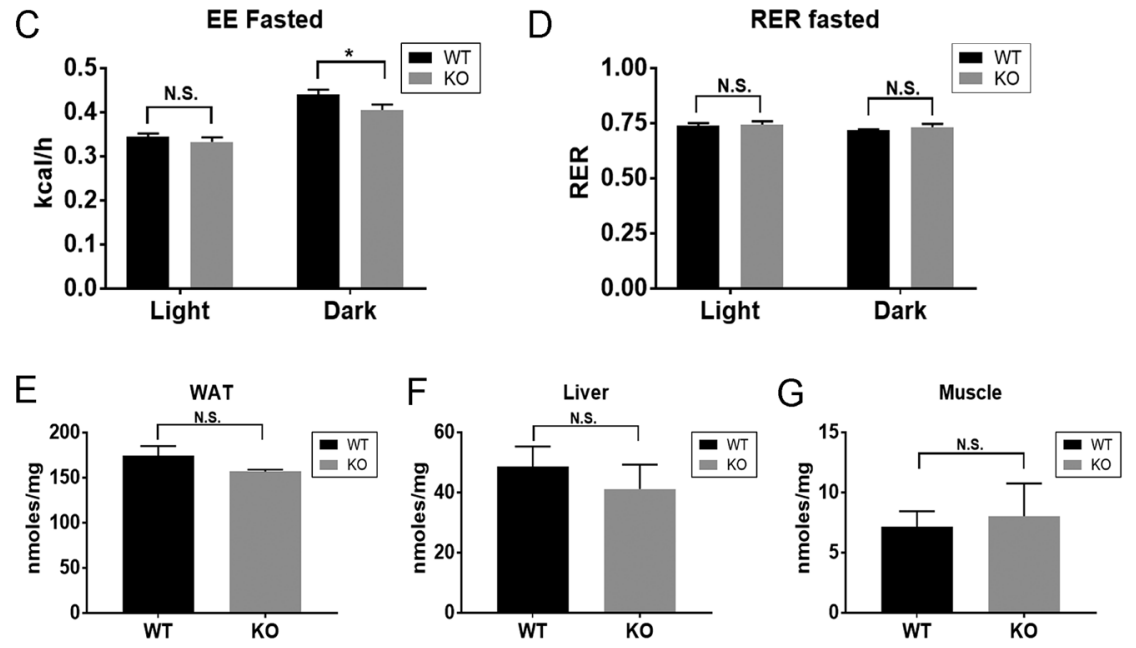

Figure 6

Metabolic responses to fasting. Female mice were single-housed in the metabolic cages for 24-36 $\mathrm{h}$ and food was removed at 8:00 for 24h. (A) Average $\mathrm{VO}_{2}$ consumption, (B): physical activity, (C) energy expenditure, (D) respiratory exchange ratio. (E, F, and G) Triacylglycerol content in tissues of fasted female mice. (E) White adipose tissue, (F) liver, (G) gastrocnemius muscle ( $n=4-12$ ) Light: 8:30-19:00pm, dark: 19:00-7:00 $(n=9, * P<0.05, * * P<0.01$, N.S. not significant by two-way ANOVA and Fisher's LSD test). 


\section{Declaration of interest}

The authors declare that there is no conflict of interest that could be perceived as prejudicing the impartiality of the research reported.

\section{Funding}

Funded by CA191653 (I P), CA197713 (A J G), CA067166 (A J G and N C D) and DK105109 (K I S). The OSUCCC shared resources are supported by a Cancer Center Support Grant (CA016058). NIH had no role in the study design, data generation, the writing of this report or the decision to submit it for publication.

\section{References}

Bal NC, Maurya SK, Sopariwala DH, Sahoo SK, Gupta SC, Shaikh SA, Pant M, Rowland LA, Bombardier E, Goonasekera SA, et al. 2012 Sarcolipin is a newly identified regulator of muscle-based thermogenesis in mammals. Nature Medicine 18 1575-1579. (doi:10.1038/nm.2897)

Beller M, Sztalryd C, Southall N, Bell M, Jackle H, Auld DS \& Oliver B 2008 COPI complex is a regulator of lipid homeostasis. PLoS Biology 6 e292. (doi:10.1371/journal.pbio.0060292)

Bensaad K, Favaro E, Lewis CA, Peck B, Lord S, Collins JM, Pinnick KE, Wigfield S, Buffa FM, Li JL, et al. 2014 Fatty acid uptake and lipid storage induced by HIF-1alpha contribute to cell growth and survival after hypoxia-reoxygenation. Cell Reports 9 349-365. (doi:10.1016/j. celrep.2014.08.056)

Cannon B \& Nedergaard J 2004 Brown adipose tissue: function and physiological significance. Physiological Reviews 84 277-359. (doi:10.1152/physrev.00015.2003)

Cannon B \& Nedergaard J 2011 Nonshivering thermogenesis and its adequate measurement in metabolic studies. Journal of Experimental Biology 214 242-253. (doi:10.1242/jeb.050989)

Denko N, Schindler C, Koong A, Laderoute K, Green C \& Giaccia A 2000 Epigenetic regulation of gene expression in cervical cancer cells by the tumor microenvironment. Clinical Cancer Research 6 480-487.

Dijk W, Mattijssen F, de la Rosa Rodriguez M, Loza Valdes A, Loft A, Mandrup S, Kalkhoven E, Qi L, Borst JW \& Kersten S 2017 Hypoxia-inducible lipid droplet-associated (HILPDA) is not a direct physiological regulator of lipolysis in adipose tissue. Endocrinology 158 1231-1251. (doi:10.1210/en.2016-1809)

DiStefano MT, Danai LV, Roth Flach RJ, Chawla A, Pedersen DJ, Guilherme A \& Czech MP 2015 The lipid droplet protein hypoxiainducible gene 2 promotes hepatic triglyceride deposition by inhibiting lipolysis. Journal of Biological Chemistry 290 15175-15184. (doi:10.1074/jbc.M115.650184)

DiStefano MT, Roth Flach RJ, Senol-Cosar O, Danai LV, Virbasius JV, Nicoloro SM, Straubhaar J, Dagdeviren S, Wabitsch M, Gupta OT, et al. 2016 Adipocyte-specific hypoxia-inducible gene 2 promotes fat deposition and diet-induced insulin resistance. Molecular Metabolism 5 1149-1161. (doi:10.1016/j.molmet.2016.09.009)

Elvidge GP, Glenny L, Appelhoff RJ, Ratcliffe PJ, Ragoussis J \& Gleadle JM 2006 Concordant regulation of gene expression by hypoxia and 2-oxoglutarate-dependent dioxygenase inhibition: the role of HIF-1alpha, HIF-2alpha, and other pathways. Journal of Biological Chemistry 281 15215-15226. (doi:10.1074/jbc.M511408200)

Fujimoto T \& Parton RG 2011 Not just fat: the structure and function of the lipid droplet. Cold Spring Harbor Perspectives in Biology 3 pii a004838. (doi:10.1101/cshperspect.a004838)

Gimm T, Wiese M, Teschemacher B, Deggerich A, Schodel J, Knaup KX, Hackenbeck T, Hellerbrand C, Amann K, Wiesener MS, et al. 2010 Hypoxia-inducible protein 2 is a novel lipid droplet protein and a specific target gene of hypoxia-inducible factor-1. FASEB Journal $\mathbf{2 4}$ 4443-4458. (doi:10.1096/fj.10-159806)

Goodman JM 2008 The gregarious lipid droplet. Journal of Biological Chemistry 283 28005-28009. (doi:10.1074/jbc.R800042200)

Granneman JG, Moore HP, Krishnamoorthy R \& Rathod M 2009 Perilipin controls lipolysis by regulating the interactions of AB-hydrolase containing 5 (Abhd5) and adipose triglyceride lipase (Atgl). Journal of Biological Chemistry 284 34538-34544. (doi:10.1074/jbc.M109.068478)

Greenberg AS, Egan JJ, Wek SA, Garty NB, Blanchette-Mackie EJ \& Londos C 1991 Perilipin, a major hormonally regulated adipocyte-specific phosphoprotein associated with the periphery of lipid storage droplets. Journal of Biological Chemistry 266 11341-11346.

Griggio MA 1982 The participation of shivering and nonshivering thermogenesis in warm and cold-acclimated rats. Comparative Biochemistry and Physiology-Part A 73 481-484. (doi:10.1016/03009629(82)90189-X)

Guo Y, Walther TC, Rao M, Stuurman N, Goshima G, Terayama K, Wong JS, Vale RD, Walter P \& Farese RV 2008 Functional genomic screen reveals genes involved in lipid-droplet formation and utilization. Nature 453 657-661. (doi:10.1038/nature06928)

Hsieh K, Lee YK, Londos C, Raaka BM, Dalen KT \& Kimmel AR 2012 Perilipin family members preferentially sequester to either triacylglycerol-specific or cholesteryl-ester-specific intracellular lipid storage droplets. Journal of Cell Science 125 4067-4076. (doi:10.1242/jcs.104943)

Khor VK, Ahrends R, Lin Y, Shen WJ, Adams CM, Roseman AN, Cortez Y, Teruel MN, Azhar S \& Kraemer FB 2014 The proteome of cholesterylester-enriched versus triacylglycerol-enriched lipid droplets. PLOS ONE 9 e105047. (doi:10.1371/journal.pone.0105047)

Krahmer N, Hilger M, Kory N, Wilfling F, Stoehr G, Mann M, Farese RV Jr \& Walther TC 2013 Protein correlation profiles identify lipid droplet proteins with high confidence. Molecular and Cellular Proteomics 12 1115-1126. (doi:10.1074/mcp.M112.020230)

Mattijssen F, Georgiadi A, Andasarie T, Szalowska E, Zota A, KronesHerzig A, Heier C, Ratman D, De Bosscher K, Qi L, et al. 2014 Hypoxia-inducible lipid droplet-associated (HILPDA) is a novel peroxisome proliferator-activated receptor (PPAR) target involved in hepatic triglyceride secretion. Journal of Biological Chemistry 289 19279-19293. (doi:10.1074/jbc.M114.570044)

Maurya SK, Bal NC, Sopariwala DH, Pant M, Rowland LA, Shaikh SA \& Periasamy M 2015 Sarcolipin is a key determinant of the basal metabolic rate, and its overexpression enhances energy expenditure and resistance against diet-induced obesity. Journal of Biological Chemistry 290 10840-10849. (doi:10.1074/jbc.M115.636878)

Qiu B, Ackerman D, Sanchez DJ, Li B, Ochocki JD, Grazioli A, Bobrovnikova-Marjon E, Diehl JA, Keith B \& Simon MC 2015 HIF2alpha-dependent lipid storage promotes endoplasmic reticulum homeostasis in clear-cell renal cell carcinoma. Cancer Discovery 5 652-667. (doi:10.1158/2159-8290.CD-14-1507)

Reue K 2011 A thematic review series: lipid droplet storage and metabolism: from yeast to man. Journal of Lipid Research $\mathbf{5 2}$ 1865-1868. (doi:10.1194/jlr.E020602)

Rowland LA, Bal NC \& Periasamy M 2015 The role of skeletal-musclebased thermogenic mechanisms in vertebrate endothermy. Biological Reviews of the Cambridge Philosophical Society 90 1279-1297. (doi:10.1111/brv.12157)

Silva JE 2011 Physiological importance and control of non-shivering facultative thermogenesis. Frontiers in Bioscience 3 352-371. (doi:10.2741/s156)

Smith WS, Broadbridge R, East JM \& Lee AG 2002 Sarcolipin uncouples hydrolysis of ATP from accumulation of $\mathrm{Ca} 2+$ by the Ca2+-ATPase of skeletal-muscle sarcoplasmic reticulum. Biochemical Journal $\mathbf{3 6 1}$ 277-286. (doi:10.1042/bj3610277)

Sztalryd C \& Kimmel AR 2014 Perilipins: lipid droplet coat proteins adapted for tissue-specific energy storage and utilization, and lipid cytoprotection. Biochimie 96 96-101. (doi:10.1016/j.biochi.2013.08.026) http://joe.endocrinology-journals.org

DOI: $10.1530 / J O E-17-0289$
(C) 2017 Society for Endocrinology Printed in Great Britain 
Sztalryd C, Xu G, Dorward H, Tansey JT, Contreras JA, Kimmel AR \& Londos C 2003 Perilipin A is essential for the translocation of hormone-sensitive lipase during lipolytic activation. Journal of Cell Biology 161 1093-1103. (doi:10.1083/jcb.200210169)

Wang V, Davis DA, Haque M, Huang LE \& Yarchoan R 2005 Differential gene up-regulation by hypoxia-inducible factor1alpha and hypoxia-inducible factor-2alpha in HEK293T cells.
Cancer Research 65 3299-3306. (doi:10.1158/0008-5472.

CAN-04-4130)

Wang X, Bjorklund S, Wasik AM, Grandien A, Andersson P, Kimby E, Dahlman-Wright K, Zhao C, Christensson B \& Sander B 2010 Gene expression profiling and chromatin immunoprecipitation identify DBN1, SETMAR and HIG2 as direct targets of SOX11 in mantle cell lymphoma. PLOS ONE 5 e14085. (doi:10.1371/journal.pone.0014085)

Received in final form 17 July 2017

Accepted 24 July 2017

Accepted preprint published online 24 July 2017
() 2017 Society for Endocrinology Printed in Great Britain
Published by Bioscientifica Ltd 